\title{
Electromagnetic Properties Performance of MWCNTs/Polyester Composites in X-band
}

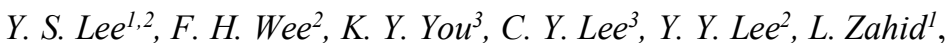 \\ Nur Hidayah Ramli ${ }^{1}$, M. S. Shakhirul ${ }^{1}$, Nurulbariah Idris ${ }^{l}$, E. M. Cheng ${ }^{4}$ \\ ${ }^{1}$ Department of Electronic Engineering Technology, Faculty of Engineering Technology, \\ Universiti Malaysia Perlis (UniMAP), Perlis, Malaysia. \\ ${ }^{2}$ Bioelectromagnetics Research Group (BioEM), School of Computer and Communication Engineering, \\ Universiti Malaysia Perlis (UniMAP), Perlis, Malaysia. \\ ${ }^{3}$ Department of Communication Engineering, Faculty of Electrical Engineering, Universiti Teknologi Malaysia (UTM), \\ 81310 UTM Johor Bahru, Johor Darul Ta'zim, Malaysia. \\ ${ }^{4}$ School of Mechatronic Engineering, Universiti Malaysia Perlis (UniMAP), Pauh Putra Campus, 02600 Arau, Perlis, Malaysia.
}

\begin{abstract}
In this paper presents nano-composites based on polyester (PE) matrix with multi-walled carbon nanotubes (MWCNTs) as fillers have been developed for microwave absorbing materials. The MWCNTs/PE composite samples were fabricated with different weight ratio of MWCNTs (3 wt $\%, 5 \mathrm{wt} \%$, and $10 \mathrm{wt} \%$ ). The electromagnetic properties of different MWCNTs/PE composite have been determined by using rectangular waveguide technique and Agilent material measurement software. Moreover, the reflection loss (microwave absorption) of MWCNTs/PE composite have been calculated based on the basis of transmission line theory. The performance of electromagnetic properties and microwave absorption of MWCNTs/PE composite were analyzed in X-band frequency. The dielectric loss properties of the composite are increse with increasing in MWCNTs weight ratio. The microwave absorption results show that such $3 \mathrm{wt} \%$ MWCNTs/PE composites sample with $4 \mathrm{~mm}$ thickness has achieved less than $-10 \mathrm{~dB}$ values $(<90 \%$ microwave absorption) of reflection loss.
\end{abstract}

\section{Introduction}

The rapid growth in science and wireless technology towards gigahertz frequency applications in modern communication has increased the electromagnetic interference (EMI) [1], [2]. EMI is cause by uncontrolled interference of electronic devices or systems. Therefore, the efficiency of EMI shielding or microwave absorbing materials (MAMs) were increasing to control the EMI pollution [3], [4]. For MAMs, it should can absorb microwave energy incident on it and have minimum reflection of the microwave energy. MAMs can use to protect electronic device and also human body from harmful effect of these microwaves energy.

Carbon nanotubes (CNTs) is a high carbon dielectric material which exhibit excellent electronic, mechanical, and material sciences [5], [6]. A composite material can defined as combining two or more materials to give a unique combination of properties. Recent years, composites based on CNTs with polymer matrix are widely used in many application due to the unique and advantageous properties offered by the new composites materials. Multi-walled carbon nanotube (MWCNT) is a tube with many CNTs inside which could be concentric or spirally spread [7]. The real part of dielectric properties is known as dielectric constant which indicate the energy store. Dielectric loss factor is the imginary part of the dielectric properties show the ability of material convert the stored energy to heat and dissipate. Therefore, the dielectric properties $\left(\varepsilon_{r}=\varepsilon_{r}^{\prime}-j \varepsilon_{r}^{\prime \prime}\right)$ of the composites is important for microwave absorber [8], [9]. The interaction of microwave energy with MAMs is microwave energy stored (absorption) in materials and convert the energy to heat.

A good MAMs are require high microwave absorption performance, thin, and light weight. The electromagnetic properties of samples were investigated using network analyzer, and measurements were carried out using the transmission line technique to determine the dielectric properties of MWCNTs/PE samples. Furthermore, the reflection loss (microwave absorption) of the samples were calculated and analyzed.

\section{Reflection Loss}

In the present study, the reflection loss of the samples were calculated based on the basis of transmission line threory to analyze the best performance in microwave absorption over 8.2- $12.4 \mathrm{GHz}$ frequency. The result can be explained base on the impendance matching as equation (1) and (2). In general, researchers use a perfect electric conductor (PEC) as a metal back plate to measure reflection loss of the MAMs [10], [11]. Figure 1 shows the illustration of the MAMs with metal backed plate.

\footnotetext{
* Corresponding author: yslee@,unimap.edu.my
} 


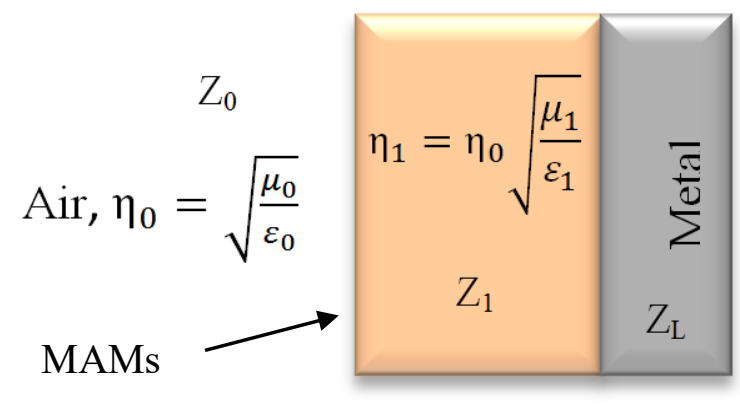

Fig. 1. Illustration of the MAMs with metal backed plate.

MAMs are functional materials that absorb microwave energy with minimum reflection and maximum attenuation/absorption of the microwave energy. The reflection of microwave energy occurs when incident energy reflected from the MAMs interface and from the metal backing of the MAMs. Input impedance $\left(Z_{\text {in }}\right)$ of MAMs close to air $(377 \mathrm{ohm})$, the reflection at the interface can be minimized. When an electromagnetic wave is incident normally to a single layer microwave absorber material is backed a metal back plate, the impedance is given by [12];

$$
Z_{\text {in }}=Z_{0} \tanh \left(j \frac{2 \pi}{c} \sqrt{\mu r \varepsilon r} f d\right)
$$

where, $Z_{0}$ is the free space impedance given by, $Z_{0}=$ $\sqrt{\mu_{0} / \varepsilon_{0}}=377 \mathrm{ohm} ; c, f$, and $d$ are the propagation velocity of the wave in free space, frequency of the incident wave, and thickness of the MAMs, respectively. The material properties namely the dielectric properties, $\varepsilon_{r}=\varepsilon_{r}^{\prime}-j \varepsilon_{r}^{\prime \prime}$ and magnetic properties, $\mu_{r}=\mu_{r}^{\prime}-j \mu_{r}^{\prime \prime}$; $d$ is the thickness of MAMs; $c$ and $f$ are the velocity of light in vacuum and the frequency of microwaves in free space, respectively. For dielectric MAMs, the permeability, $\mu_{r} \approx 1-\mathrm{j} 0$.

In case of a metal backed single layered absorber, the normalized input impedance with respect to impedance in free space and the reflection loss with respect to the normal incident plane wave are given by [13];

$$
\mathrm{R}_{\mathrm{L}}(\mathrm{dB})=-20 \log _{10}\left[\frac{(\mathrm{Zin}-1)}{(\mathrm{Zin}+1)}\right]
$$

There have two reflection phenomenon occurs when the microwave energy interact with MAMs. The first reflection is at the air-absorber interface while the another reflection is the energy propagated through the materials and bounce back from the metal backing of the MAMs. Reflection at the interface can be minimized by making input impedance of MAMs close to that of free space as known as impedance matching.

\section{Experimental}

The multi-walled carbon nanotubes (MWCNTs) used in this experiment was purity $99 \mathrm{wt} \%$ purity. MWCNTs is a nanomaterial which very hard to compacted due to the very large surface area. Hence, MWCNTs in this experiement was composites with polymer (polyester resins). In this experiment, the PE resins and Methyl ethyl ketone peroxide (MEKP) harden agent weight ratio are constants. The different weight ratio of MWCNTs as filler is the main parameter in this study. The MWCNTs/PE composites are prepared using mechanical mixing and MEKP is used as hardent agent for MWCNTs/PE samples. The samples are prepared in different weight ratio of MWCNTs $(2 \mathrm{wt} \%, 3 \mathrm{wt} \%$, and $5 \mathrm{wt} \%$ ) with dimension $22.860 \mathrm{~mm} \times 10.160 \mathrm{~mm} \times 5$ $\mathrm{mm}$. The MWCNTs/PE composites preparation process was mixed the MWCNTs with PE resins and stir for 1 hour shown in Figure 2. Then, the composites of the MWCNTs/PE was mixed with MEKP and poured into the rectangular sample holder mould flange and cured in room temperature $25{ }^{\circ} \mathrm{C}$ for 24 hours. The different rectangular samples of MWCNTs/PE was fabricated. Figure 3 shows the fabricated rectangular samples of MWCNTs/PE.

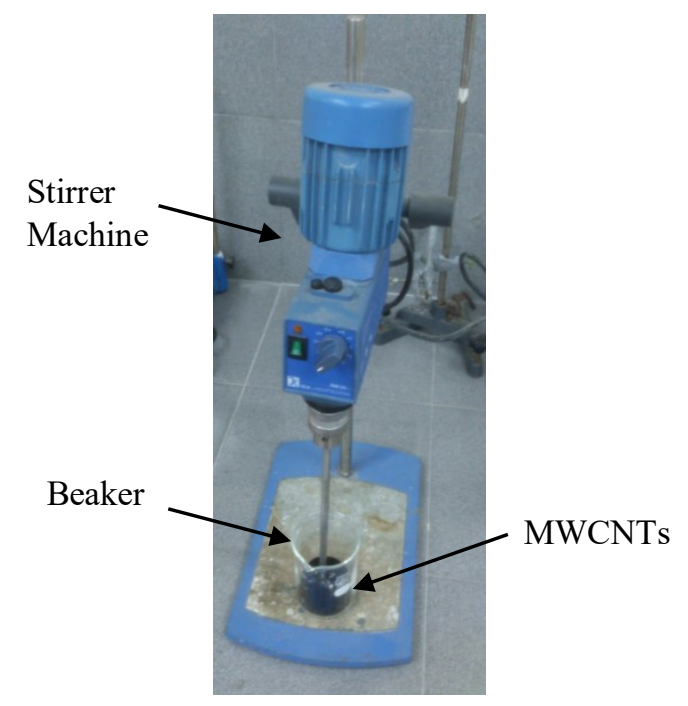

Fig. 2. The MWCNTs composites is stired with PE resins.

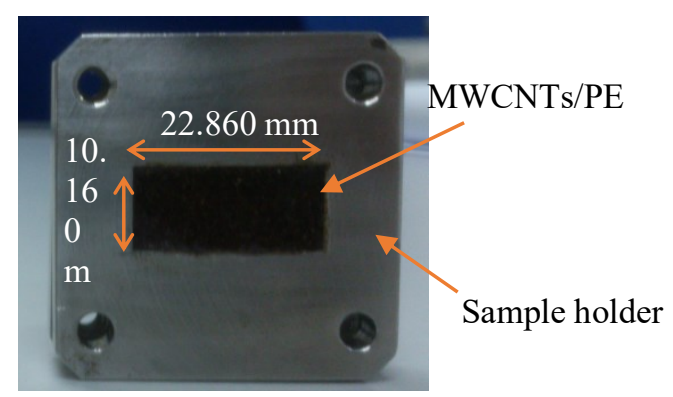

Fig. 3. MWCNTs/PE rectangular samples placed inside the sample holder.

\subsection{Material Properties Measurement}


The electromagnetic properties of MWCNT/PE nanocomposites with various weight percentage MWCNTs were first measured in the X-band to get an indication of dielectric properties dependence on MWCNT percentage. There have two methods common use in the measure electromagnetic properties of materials; free space method and transmission line method. In free space method, the materials propertis characterization much more flexible to measure under dfferent condition. But the material under test (MUT) required larger than the horn antennas. In a transmission line method, the MUT is placed in a a piece of transmission line or sample holder. The MUT are placed in samples holder between two waveguide adaptors. This method is widely use in measure electromagnetic properties of small samples. The electromagnetic properties of material are characterize based on the basis of the reflection from the MUT and transmission through the MUT [14]. In this study, transmission line method is used to measure the dielectric properties of materials which suitable for small samples.

The rectangular waveguide transmission line technique is used to measure the dielectric properties of the MWCNTs/PE sample. A pair of coaxial cables were connected between Agilent network analyzer model E8362B and the rectangular waveguide adaptors (WR90). The fabricated MWCNTs/PE samples are inserted into the rectangular sample holder as shown in Figure 4. The sample holder is well connected between two rectangular waveguide adaptors with screws as shown in Figure 4. Figure 5 shows the sample holder is custom manufacture with size $a=22.860 \mathrm{~mm}, \mathrm{~b}=10.160 \mathrm{~mm}$, and $\mathrm{d}$ (thickness) $=4 \mathrm{~mm}$.

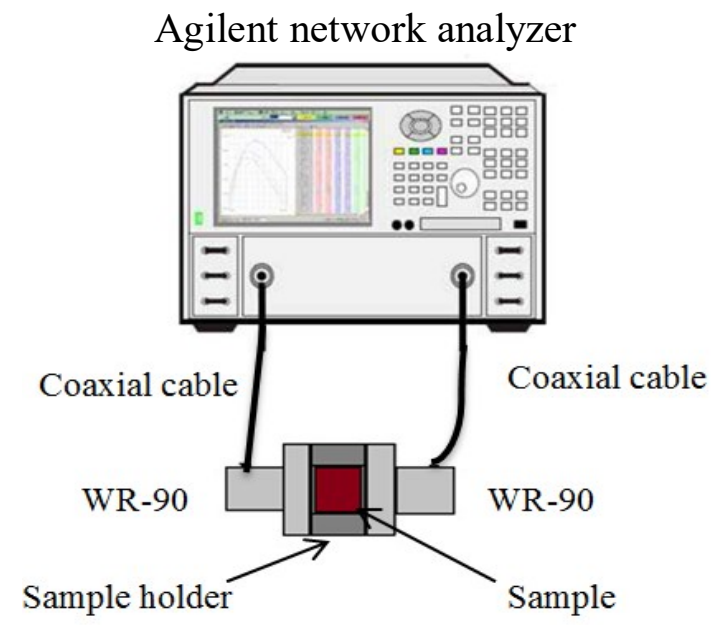

Fig. 4. Rectangular waveguide transmission line technique measurement setup.

Figure 5 shows the Thru-Reflect-Line (TRL) calibration kits of the rectangular waveguide transmission line. The TRL calibration of the measurement is to eliminate the testing error induced by the gap between sample and flange. The transmission line is enclosured with conductor material. Therefore, the microwave just can propagated inside the rectangular waveguide. Then, The dielectric properties of MWCNTs/PE samples are computed by using the Agilent software 85071E [8].

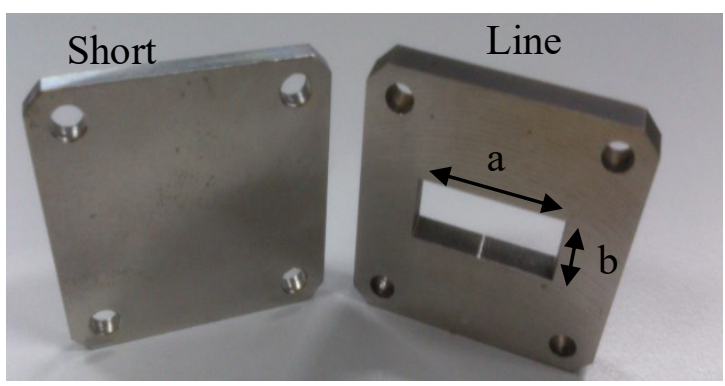

Fig. 5. TRL calibration Line and short for rectangular waveguide.

\section{Results and Discussion}

Figure 6 (a) and (b) represent the dielectric constant, loss factor, and loss tangent of different MWCNTs filler $3 \mathrm{wt} \%, 5 \mathrm{wt} \%$, and $10 \mathrm{wt} \%$ respectively. The dielectric properties of MWCNTs/PE samples are measured using rectangular waveguide mehod in X-band frequency band. From the dielectric properties results, it can be observed that the weight percentage of MWCNTs filler effect the dielectric constant, loss factor, and loss tangent of dielectric properties. The dielectric properties increase with increasing of wt. \% MWCNTs filler over $8.2 \mathrm{GHz}$ to $12.4 \mathrm{GHz}$. The dielectric constant values increase from 4.08 to 15.52 with increasing the MWCNTs filler from $3 \mathrm{wt} \%$ to $10 \mathrm{wt} \%$. Similar to the loss factor values increase from 1.63 to 11.27 . For the lower MWCNT weight percentages, the loss factor was relatively flat with frequency. Table 1 shows the average values of dielectric properties of MWCNTs/PE samples in Xband.

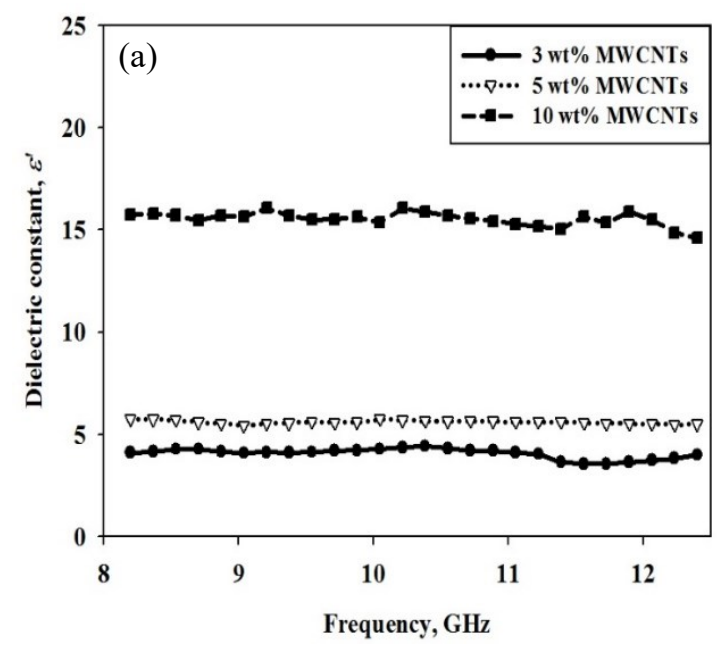




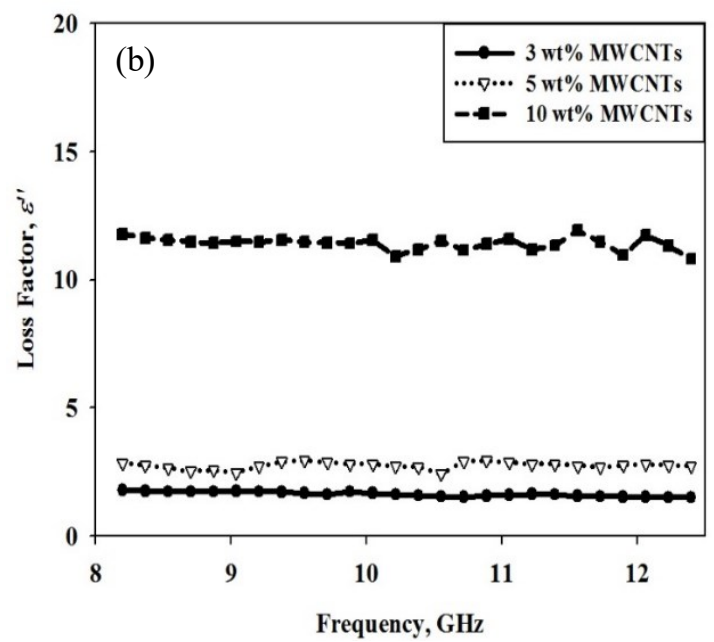

Fig. 6. (a) Dielectric constant and (b) loss factor of $3 \mathrm{wt} \%, 5$ $\mathrm{wt} \%$, and $10 \mathrm{wt} \%$ MWCNTs fillers (wt $\%$ ).

Table 1. The dielectric constant and loss factor of MWCNTs/PE samples in X-band.

\begin{tabular}{|c|c|c|}
\hline \multirow{2}{*}{ Sample } & \multicolumn{2}{|c|}{ Average values } \\
\cline { 2 - 3 } & Dielectric constant & Loss factor \\
\hline $\mathbf{3} \%$ MWCNTs & 4.08 & 1.63 \\
\hline $\mathbf{5 \%}$ MWCNTs & 5.61 & 2.72 \\
\hline $\mathbf{1 0} \%$ MWCNTs & 15.52 & 11.27 \\
\hline
\end{tabular}

From the calculated reflection loss in Figure 7, a broadband MAMs would have a MWCNTs weight percentage 3 wt $\%$ of the composite. The results for 5 wt $\%$ MWCNT samples cover the X-band with less than $10 \mathrm{~dB}(<90 \%$ microwave absorption $)$ shown in Figure 7. Nevertheless, the 3 wt $\%$ MWCNTs weight percentage in MWCNTs/PE composites shows better broadband absorption than $5 \mathrm{wt} \%$ and $10 \mathrm{wt} \%$ of MWCNTs in X-band. The sample with 3 wt $\%$ MWCNTs shows average lower values of reflection loss in the frequency of X-band compare to the samples with $5 \mathrm{wt} \%$ and $10 \mathrm{wt} \%$ MWCNTs. At the frequency of 10 $\mathrm{GHz}$ the reflection loss value is close to $-15.8 \mathrm{~dB}$ which corresponds to $97 \%$ of microwave absorption. From Figure 7, observed that sample $5 \mathrm{wt} \%$ MWCNTs reflection loss increase with increasing the frequency due to the mismatch of the impedance matching $\left(Z_{\text {in }}\right)$ at higher freqeuncy. Similar problem for sample with $10 \mathrm{wt} \%$ MWCNTs, MWCNTs/PE sample with $10 \mathrm{wt} \%$ MWCNTs has the highest values of reflection loss (lowest absorption) in X-band. This shows that the sample with high dielectric properties in range, $\varepsilon_{r}{ }^{\prime}=15$ to 15.5 and $\varepsilon_{r} "=11.5$ to 12.5 with $4 \mathrm{~mm}$ thickness is not suitable use as microwave absorber in X-band. All this mismatch of impedance matching $\left(Z_{\text {in }}\right)$ over frequency range in $X$-band can be explained using the equation (1) and (2). The dielectric properties of materials and frequency range are the important factor to determine the absorption of MAMs.

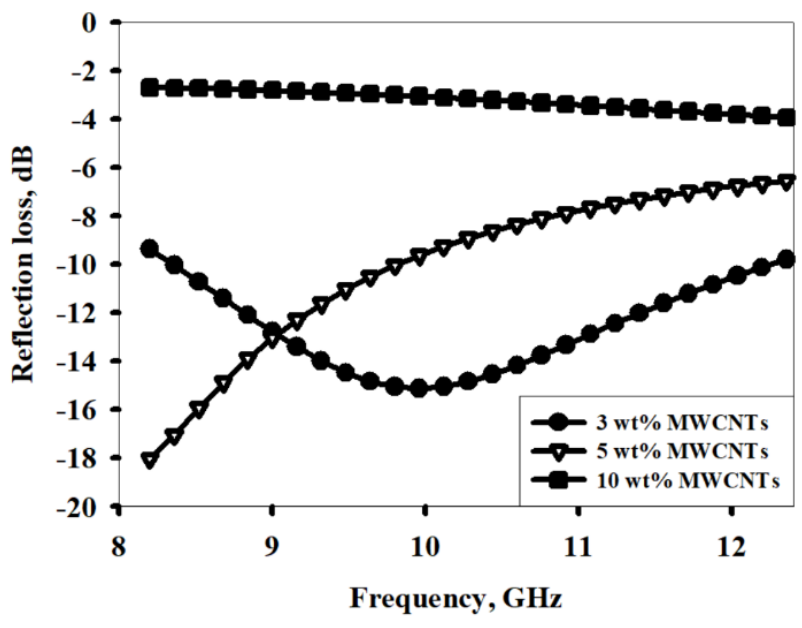

Fig. 7. The reflection loss of $3 \mathrm{wt} \%, 5 \mathrm{wt} \%$, and $10 \mathrm{wt} \%$ MWCNTs fillers (wt.\%).

\section{Conclusions}

This study shows that the different application in microwave absorber with flexibility of the dielectric properties of materials to use as MAMs. MWCNTs/PE samples with different weight ratio $(3,5,10 \mathrm{wt} \%)$ of the same MWCNTs were fabricated. The dielectric properties of MWCNTs/PE samples were measured with rectangulare transmission line technique and an Agilent $85071 \mathrm{E}$ software in the X-band frequency. The microwave absorption properties of the MWCNTs/PE samples were design and calculated based on the basis of transmission line theory.

This research was funded by the grant of Fundamental Research Grant Scheme (No. 9003-00591) under Ministry of Education, Malaysia.

\section{References}

[1] B. Archambeault, C. Brench, and S. Connor, "Review of Printed-Circuit-Board Level EMI/EMC Issues and Tools," IEEE Trans. Electromagn. Compat., vol. 52, no. 2, pp. 455-461, 2010.

[2] M. H. Al-Saleh and U. Sundararaj, "Electromagnetic interference (EMI) shielding effectiveness of PP/PS polymer blends containing high structure carbon black," Macromol. Mater. Eng., vol. 293, no. 7, pp. 621-630, 2008.

[3] I. B. Darney, Circuit Modeling for Electromagnetic Compatibility. Institution of Engineering and Technology, 2013.

[4] P. Saini and M. Arora, "Microwave Absorption and EMI Shielding Behavior of Nanocomposites Based on 2 Intrinsically Conducting Polymers, 3 Graphene and Carbon Nanotubes 4," 2012. 
[5] T. Zhao, C. Hou, H. Zhang, R. Zhu, S. She, J. Wang, T. Li, Z. Liu, and B. Wei, "Electromagnetic wave absorbing properties of amorphous carbon nanotubes.," Sci. Rep., vol. 4, p. 5619, 2014.

[6] D. L. Zhao, X. Li, and Z. M. Shen, "Microwave absorbing property and complex permittivity and permeability of epoxy composites containing $\mathrm{Ni}$ coated and Ag filled carbon nanotubes," Compos. Sci. Technol., vol. 68, no. 14, pp. 2902-2908, 2008.

[7] C. M. Chang, J. C. Chiu, W. S. Jou, T. L. Wu, and W. H. Cheng, "New package scheme of a $2.5-\mathrm{Gb} / \mathrm{s}$ plastic transceiver module employing multiwall nanotubes for low electromagnetic interference," IEEE J. Sel. Top. Quantum Electron., vol. 12, no. 5, pp. 10251030, 2006.

[8] Y. S. Y. Lee, M. M. F. B. A. Malek, E. M. E. E. M. Cheng, W. W. Liu, K. Y. You, M. N. Iqbal, F. H. Wee, S. F. Khor, L. Zahid, and M. F. bin Haji Abd Malek, "Experimental determination of the performance of rice husk-carbon nanotube composites for absorbing microwave signals in the frequency range of 12.4-18 GHz," Prog. Electromagn. Res., vol. 140, no. April, pp. 795-812, 2013.

[9] D. A. Makeiff and T. Huber, "Microwave absorption by polyaniline-carbon nanotube composites," Synth. Met., vol. 156, no. 7-8, pp. 497-505, 2006.
[10] S. Das, G. C. Nayak, S. K. Sahu, P. C. Routray, A. K. Roy, and H. Baskey, "Microwave absorption properties of double-layer composites using $\mathrm{CoZn} / \mathrm{NiZn} / \mathrm{MnZn}$-ferrite and titanium dioxide," $J$. Magn. Magn. Mater., vol. 377, pp. 111-116, 2015.

[11] J. W. Kim and S. S. Kim, "Microwave absorbers of two-layer composites laminate for wide oblique incidence angles," Mater. Des., vol. 31, no. 3, pp. 1547-1552, 2010.

[12] A. Ghasemi, A. Paesano, and C. F. C. MacHado, "Magnetic and reflection loss characteristics of terbium substituted cobalt ferrite nanoparticles/functionalized multi-walled carbon nanotube," in IEEE Transactions on Magnetics, 2012, vol. 48, no. 4, pp. 1528-1531.

[13] S. Ozah and N. Bhattacharyya, "Nanosized barium hexaferrite in novolac phenolic resin as microwave absorber for X-band application," J. Magn. Magn. Mater., vol. 342, pp. 92-99, Sep. 2013.

[14] Y. S. Lee, F. Malek, E. M. Cheng, W. W. Liu, K. Y. You, K. Y. Wee, L. Zahid, and H. A. Rahim, "Single layer microwave absorber based on rice husk-mwents composites," ARPN J. Eng. Appl. Sci., vol. 11, no. 14, pp. 8932-8937, 2016. 